\title{
Proyectos de aula, una experiencia exitosa de investigación formativa
}

\author{
Helga Ofelia Dworaczek Conde ${ }^{1}$
}

\begin{abstract}
Resumen
El presente artículo, utiliza la antropología y sociología como marco teórico, crea un diagrama metodológico a partir de los planteamientos de Alfred Reginald, Radcliffe-Brown, Bronislaw Malinowsky y Émile Durkheim, determinando la importancia de la investigación contextual desde el planteamiento de los Toffler en: La revolución de la riqueza, presentando la evolución de la sociedad económica desde la innovación, tecnología e investigación.

Se incluye el análisis del Dr. Ricardo Mosquera desde la perspectiva de educación globalizada, retomando la propuesta pedagógica Agustiniana, ilustrando experiencias en proyectos de aula, concluyéndolas con los logros obtenidos en el proceso cognitivo, competencial y de consolidación de valores en la enseñanza -aprendizaje de estudiantes, docentes y administrativos como una red social del conocimiento.
\end{abstract}

Palabras clave: Jóvenes investigadores, métodos de enseñanza, proceso de aprendizaje. 


\title{
Classroom projects, a successful experience of formative research
}

\begin{abstract}
This paper seeks to provide a theoretical framework based on sociology and anthropology and to make a methodological scheme using the approaches by Alfred Reginald Radcliffe-Brown, Bronislaw Malinowsky and Émile Durkheim; capturing also the importance of the contextualized research through the perspective view displayed by the Tofflers in Revolutionary Wealth, and thus revealing the evolution of the economic society.

From a globalized education approach, the study made by the professor Ricardo Mosquera has also been joint, retaking the Augustinian pedagogical proposal, which illustrates educational experiences such as classroom projects, the achievements made in the cognitive and skilling process, and the reinforcement of values in the teachinglearning process of students, teachers and administrative officers as a social network of knowledge.
\end{abstract}

Keywords: Young researchers, learning process, teaching methods.

Recibido: 29/07/2014

Aceptado: 03/10/2014

\section{Introducción}

El presente artículo crea un diagrama metodológico a partir de los planteamientos de Radcliffe-Brown, retoma el funcionalismo estructuralista de Malinowsky; describe y analiza la cultura desde una experiencia personal y el estudio de la educación como fenómeno social desde Émile Durkheim, quien plantea que la educación crea un hombre completamente nuevo como agente positivo a una sociedad. Los anteriores planteamientos permiten determinar la importancia de la investigación en un contexto mundial. Así, con el planteamiento de Alvin y Heidi Toffler, en su libro La revolución de la riqueza, se presenta la evolución de la sociedad en un contexto económico desde la innovación, la tecnología y la investigación. Igualmente, en este contexto de globalización, es necesario revisar la literatura referente a la educación, por lo que se incluye el análisis del Dr. Ricardo Mosquera, quien afirma que no es suficiente contar o 
tener abundante acceso a los recursos naturales, sino que es más importante poseer el conocimiento para manejarlos, gestionarlos y utilizarlos de la mejor manera posible sin causar daños irreversibles al medio ambiente. Finalmente se retoma la propuesta pedagógica Agustiniana y se presentan las experiencias en proyectos de aula para concluir con los logros obtenidos en el proceso cognitivo, de competencias y de consolidación de valores en la enseñanza - aprendizaje de estudiantes, docentes y administrativos como una red social del conocimiento.

\section{Problema que aborda el proyecto}

¿Cuál es el aporte de la investigación formativa al proceso integral de enseñanza -aprendizaje?

\section{Metodología}

La metodología del presente artículo se sustenta en el método hipotético deductivo, que corresponde a una descripción del método científico y parte de la observación repetida de fenómenos comparables, y en el cual las leyes generales se deducen. Dentro de los pasos establecidos para desarrollar este método se encuentran la observación (experiencia, proceso empírico), el planteamiento de hipótesis (experiencia, proceso empírico), las deducciones de conclusiones a partir de conocimientos previos y el volver a la inducción para su verificación.

\section{Marco teórico}

Para dar respuesta a la pregunta problémica se utilizan como herramientas teóricas los planteamientos de Alfred R. Radcliffe-Brown (1975), quien afirmó que los elementos de una sociedad son interdependientes entre sí (funcionalismo estructuralista), y Bronislaw Malinowsky (1978), autor que basa su trabajo en la experiencia personal desde una visión funcional de la cultura compuesta por la descripción y el análisis.

Por otra parte, Emile Durkheim (Chinoy, 1990), al analizar la sociología moderna e identificar los hechos sociales como modos de actuar, pensar y sentir, externos al individuo y que poseen un poder de coerción en virtud del cual se impone la consciencia colectiva, determinada por el volumen, intensidad, rigidez y contenido de las ideas que unen a una sociedad. Esta consciencia colectiva existe antes del nacimiento, actúa dentro y fuera de la sociedad con carácter coercitivo.

Retomando el documento, «La educación como fenómeno social» de Émile Durkheim (2012), quien define que la educación, lejos de tener simplemente por fin desarrollar al hombre tal como sale de las manos de la naturaleza, tiene por objeto extraer de allí un hombre enteramente nuevo; crea un ser que no existe, salvo en el estado de germen indiscernible: el ser social. Ella nos enseña a dominarnos, a constreñirnos; es también, siguiendo sus necesidades, que se 
decide la cantidad y naturaleza de los conocimientos que debe recibir el niño, y lo mismo a través de ella, que se conserva la ciencia adquirida por las generaciones anteriores, también la que transmite a las nuevas generaciones. Forma en nosotros todo lo que supera la esfera de las puras sensaciones: nuestra voluntad como nuestro entendimiento son moldeados a su imagen. Este planteamiento permite dar una base sociológica al proceso de enseñanza-aprendizaje como hilo conductor de la construcción de la sociedad del siglo XXI, la cual corresponde a características particulares de acuerdo a las necesidades del entorno interno y externo.

Con los anteriores planteamientos teóricos se crea un diagrama de flujo que permite dar respuesta a la pregunta planteada.

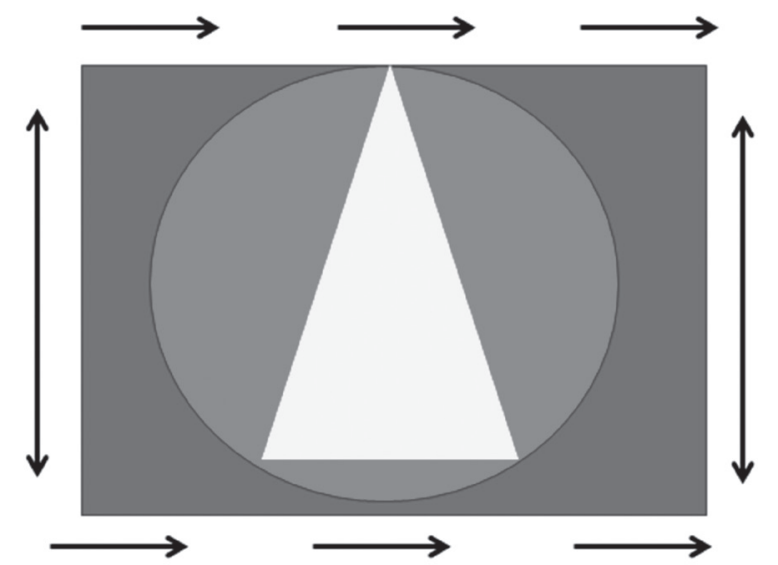

Gráfico 1. Diagrama teorías sociológicas que explican la investigación formativa

\section{Cuadro de conversión}

Experiencia personal. Visión funcional $\triangle$

Consciencia colectiva $\bigcirc$

Interdependencia en la sociedad

Etapas de desarrollo (niño, pre-adolescente, adolescente, joven, adulto, adulto mayor)

Entorno interno y externo

El diagrama anterior representa la conexión entre las teorías y la experiencia práctica académica en investigación formativa, donde la experiencia personal de los estudiantes y docentes representan la visión funcional que permite un análisis y descripción de los factores que responden la pregunta problémica planteada en el proyecto de aula.

También la consciencia colectiva que actúa según el volumen, la intensidad, la rigidez y el contenido da paso al trabajo en equipo por un objetivo en común fortaleciendo esta competencia del ser y hacer. 
Así, los seres humanos por su naturaleza de ser sociales, se caracterizan por la interdependencia en contexto, donde los estudiantes y docentes comparten saberes y trabajan cooperativamente potencializando el aprendizaje en ambas direcciones. El individuo presenta un desarrollo evolutivo (niño, adolescente, joven, adulto, adulto mayor) dando diferentes respuestas y perspectivas a un mismo planteamiento, según la etapa en la que se encuentre y la relación directa e indirecta con su entorno interno y externo.

Finalmente la interacción de estos elementos permite que el trabajo interdisciplinario, interpersonal e interdependiente tenga como resultado el aprendizaje significativo.

\section{Desarrollo}

El presente documento muestra la importancia de la investigación en el proceso educativo como lo ilustra desde las perspectivas económica, social, política y cultural el texto La revolución de la riqueza de Alvin y Heidi Toffler ${ }^{2}$ (2010), escritores futuristas que explican cómo a través de la evolución del ser humano se han conformado sociedades con características políticas y culturales determinadas, siendo el factor tecnológico constante y reactivador de las relaciones comerciales.

En su libro, muestran a la riqueza como cualquier posesión que tiene una utilidad, al proporcionarnos alguna forma de bienestar por sí misma o mediante su intercambio con alguna otra forma de riqueza que satisfaga dicho bienestar. Además, tiene tres sistemas representados por olas: la primera hace referencia al período de la prehistoria con la consolidación del sedentarismo gracias al desarrollo de la agricultura y la creación de las ciudades, Estados e imperios; la segunda representa el proceso de industrialización, y la tercera sustituye los factores de producción tradicional (tierra, capital, trabajo) por la revolución del conocimiento y, más adelante, la gestión del mismo, es decir, el conocimiento como base de la riqueza.

Es así como el excedente económico de los pueblos primitivos les permitió desarrollar el intercambio de bienes (trueque), aunque si no era posible esta práctica, era común que por la fuerza se apoderaran de los factores de producción que pudieran brindarles riqueza. En el proceso histórico las grandes civilizaciones antiguas como Mesopotamia, Egipto, China, India, Grecia y Roma, así como la Maya, Azteca e Inca lograron crear monedas, unidades de medidas y contratos comerciales que regularon sus relaciones comerciales.

Luego, en la Edad Media los pueblos expandieron sus fronteras gracias a la consolidación de rutas comerciales marítimas y terrestres. En el siglo XII se consolidó la importancia de las ciudades, lo que permitió un comercio local y

2. Alvin Toffler: norteamericano doctor en letras, leyes y ciencia. Reconocido por su literatura futurista, gracias a sus estudios en tecnología, nuevos medios y sus efectos sociales. Heidi Toffler: norteamericana lingüista de la Universidad de Nueva York, escribe con su esposo (Alvin) desde 1954. 
regional (modalidad aplicada según el tipo de mercancía). Además, es importante recalcar que el comercio internacional de Occidente se desarrolló principalmente con Asia. A nivel europeo se consolidaron las ferias con lo que se incrementó el intercambio comercial entre diferentes sociedades. Luego de todos estos eventos los países que se volvieron líderes comerciales de esta época fueron Italia, Inglaterra, España, Portugal, Francia y Alemania.

En la edad moderna, el descubrimiento de América, el desarrollo de la tecnología, los cambios político-económicos (revoluciones burguesas) y la reforma protestante, generaron un gran avance en materias política, social y económica, en especial para el comercio. Así, se modificaron las estructuras de poder al consolidarse los Estados-nación, en los cuáles el monarca ya no tiene un poder absoluto y se hace un cambio a un poder constitucional donde se permite a cada ciudadano (no vasallo o siervo) decidir qué hacer con su vida y a qué dedicarse, así como la consolidación de las libertades individuales y del capitalismo. Además, la riqueza de las naciones (Adam Smith, 2012) se lograba por medio de los excedentes de oro y plata (mercantilismo) o excedentes de la agricultura (fisiocracia).

El siglo XVIII, conocido como el siglo de las luces por sus grandes avances en materia social, política, económica y cultural, fue la plataforma para la revolución industrial. Esta revolución se dio gracias a los avances legislativos en los países europeos, como es el caso de Venecia que en 1474 instauró la primera ley de patentes y de Estados Unidos que en 1790 aprobó la primera ley de patentes, en concordancia con la Constitución de Filadelfia; a esto se le suma que en el período 1790-1850 se establecieron leyes de patente industrial en muchos Estados europeos. Igualmente, Inglaterra es el primer país en contemplar la protección de los derechos de autor a través de leyes de derecho de copia (copyright) mediante la firma de la cédula real de la Reina Ana en 1709, proceso de protección de ciencia, investigación y tecnología que se extendió muy pronto por los países occidentales.

Estos avances tecnológicos permitieron aumentar significativamente la producción al reemplazar la mano de obra humana por máquinas eficientes y automatizadas. Esta revolución industrial dio paso al liberalismo económico liderado por Inglaterra bajo los planteamientos de John Locke y la economía clásica de Adam Smith y David Ricardo. En el plano político, el liberalismo encabezado por Francia con los planteamientos de Rousseau, Montesquieu y Voltaire dio las bases ideológicas del capitalismo que permitieron ampliar sus fronteras nacionales con la consolidación del capital financiero, dando origen a una nueva etapa, la globalización que tiene como agente a las multinacionales y a los organismos multilaterales.

La revolución de la riqueza se basa en tres conceptos fundamentales: el tiempo, el espacio y el conocimiento. El mayor reto para Toffler es la velocidad de los cambios, que a su vez varían rápidamente, sin embargo, la velocidad en que 
se desarrollan estos cambios en las instituciones básicas de cada país no tiene un ritmo acorde con las necesidades de la economía del conocimiento, esto comprende el primer concepto. En el segundo término, se observa que hay regiones con conocimiento intensivo que dan lugar a un valor añadido. El tercer fundamento, el conocimiento, es generador de las variaciones en el tiempo y el espacio, pues como factor principal en la reproducción de riqueza se distingue por ser intangible, relacional, sin ser lineal, se empareja con otro conocimiento, es más portátil que cualquier otro producto, puede comprimirse, se puede almacenar en espacios pequeños, puede ser explicito o implícito, expresado o no, compartido o tácito.

En conclusión, en esta obra se plantea el paso de la sociedad industrial a la tecnológica o sociedad de la economía del conocimiento, sin que se hubieran adecuado los mecanismos institucionales, económicos, fiscales, educativos y culturales acordes al nuevo cambio. También se observa que se ha creado un sistema de riqueza sin precedentes en la historia de la humanidad, una riqueza revolucionaria que permite acabar con la pobreza, pero comprueba que esto no se logra porque más que aplicar medidas nuevas lo que se hace es adaptar en extremo los antiguos modelos sin un buen resultado, puesto que no encajan y al contrario generan más problemas.

Es el modelo de pensamiento lo que retrasa que nos adecuemos al cambio de paradigma que se está produciendo: los economistas son incapaces para reconocer este cambio histórico y comprender la riqueza revolucionaria. Por supuesto hay excepciones que confirman la regla. Por las mismas razones en la obra se refiere a la quiebra de los sistemas educativos, escolares o universitarios, en casi todos los países, por obsoletos y ajenos a las nuevas formas de organización y aprendizaje de la era de la información.

Por último, frente al planteamiento de Durkheim (2012), referente a la educación, la obra expone que: la sociedad necesita personas que se ocupen de los ancianos y que sepan cómo ser compasivos y honestos. La sociedad necesita gente que trabaje en los hospitales. La sociedad necesita todo tipo de habilidades que no son solo cognitivas, son emocionales, son afectivas. No podemos montar la sociedad sobre datos. Los analfabetos del siglo XXI no serán aquellos que no sepan leer y escribir, sino aquellos que no sepan aprender, desaprender y reaprender. Introduciendo así un nuevo mundo con la nanotecnología ${ }^{3}$, aspecto muy popular en la actualidad (Vázquez, 2013).

\section{Otros estudios}

El Dr. Ricardo Mosquera (2002), plantea que en un mundo más integrado, en que la rivalidad lleva a una mayor competencia, el desarrollo de capacidades humanas y de innovación son las claves o garantes para prosperar en una 
economía globalizada. Actualmente, para ser más competitivo no es suficiente contar o tener abundante acceso a recursos naturales, es más importante poseer el conocimiento para manejarlos, gestionarlos y utilizarlos de la mejor manera posible sin causar daños irreversibles al medio ambiente. Se puede decir que hoy más que nunca el mundo vive en la era del conocimiento, pierde toda validez afirmar que la riqueza de un país está determinada solamente por sus recursos naturales (posición geográfica), el tipo de mercancías que produzca y los bienes físicos (infraestructura) con que cuente. Para el siglo XXI lo realmente importante es desarrollar los conocimientos que permitan el mejoramiento de procesos tecnológicos que den un valor agregado a los productos extraídos y producidos.

El siguiente análisis parte de la experiencia pedagógica en la Universitaria Agustiniana, en el Programa de Negocios Internacionales, para los períodos académicos 2011-II, 2012-I y 2012-II, que permite deducir que el anterior planteamiento es verdadero, el capital humano debe ser estructurado con énfasis en la investigación formativa para llegar a la aplicada. A continuación se retoma la propuesta pedagógica básica que permite determinar las características particulares del contexto donde se desarrolla el presente estudio.

Estos proyectos de aula se desarrollaron con base en «el trípode existencial de la propuesta pedagógica Agustiniana» donde se habla de formación integral en cuanto que abarca tres aspectos: lo educativo, lo trascendente (lo religioso, lo cristiano) y lo agustiniano; ello se encuentra plasmado en los proyectos educativos institucionales agustinianos. La escuela agustiniana es humanista y generadora de humanidad, crítica, participativa, liberadora, y desde esos criterios educa en valores éticos y es a partir de estos que se tienen en cuenta dos momentos fundamentales: la apertura a la vida o comienzo de la aventura y el camino hacia la madurez o la importancia de vivir:

Educar en clave agustiniana es crear una acción que despierta y capacita para leer el mensaje de los propios errores. Es enseñar a pensar y no tanto enseñar qué pensar, ya que se educa para saber vivir o vivir sabiamente, para ser auténticos, para aprender a estar y vivir la unidad, para amar, para vivir y buscar la paz, para ser creativo, libre, particip ativo, feliz y trascendente. No en vano la visión agustiniana de la educación pretende que los educandos sean protagonistas de su propio desarrollo, capaces de encontrar en su ser y su compartir el verdadero sentido del proceso educativo, de tal manera que se puedan formar jóvenes para un desarrollo constante, equitativo e integral con sentido evangélico, deseosos de participar en la transformación de su entorno. Esto implica que el educador agustiniano, consciente de la árida búsqueda de la verdad científica en la sociedad tecnológica, ha de estar dispuesto a darle a sus estudiantes algo más que una simple información: debe darles el deseo de aunar la verdad de la mente con la verdad del corazón. Eso es formación integral. 
Transparencia y no solo eficiencia. La escuela agustiniana tiene un carácter experimental y hace que el educador sea un buscador de nuevos horizontes desde una base antropológica, de por si incitante, que consiste en rehacer la existencia desde la interioridad, desde donde mejor se pueden recoger los elementos fundamentales del proceso educativo, descubriendo el espíritu que lo anima y algunas aplicaciones prácticas de las diferentes etapas del proceso (Uniagustiniana, 2012).

Como consecuencia de la práctica pedagógica Agustiniana (experiencia personal, visión funcional -descriptiva y analítica-), la experiencia dentro de las aulas de clase con los proyectos de aula e investigación, ha generado las bases para la creación del semillero de investigación Visionarios Emergentes. Este proceso se facilitó gracias a la dinámica de investigación formativa que se desarrolla en las clases como parte integral de la didáctica.

Cada proyecto contó con una identidad de grupo y espacio académico diferentes (consciencia colectiva). El primer proceso fue dentro de la asignatura política comercial y acuerdos internacionales, de cuarto semestre, en donde el promedio de la calificación fue 4,5, con 65 estudiantes. La dinámica se desarrolló en torno a un juego de roles donde cada grupo de estudiantes asumían un continente y debían presentarlo como una alternativa para la inversión (entorno interno, externo e interdependencia). En este proceso se generaron espacios específicos característicos para cada continente y se analizaron diversas variantes como historia, geografía, escenario político, económico, cultural y en especial, los beneficios en materia de acuerdos, tratados y pactos con carácter comercial. Además, presentaron un documento escrito en el que desarrollaron una metodología de aprendizaje basada en la solución de problemas y en el trabajo cooperativo. Finalmente, su actividad lúdica fue el escenario negociador típico del continente elegido con su portafolio de servicios.

En el segundo proyecto participaron 135 estudiantes de las asignaturas negocios internacionales, política comercial y acuerdos internacionales, con un promedio de calificación de 4.5 sobre 5.0. En las clases prepararon una ponencia en grupos de trabajo dentro del concurso de ponencias Carlos Lleras Restrepo, liderado por el Programa de Negocios Internacionales (experiencia personal, consciencia colectiva, interdependencia, entorno interno y externo). De esta forma, los estudiantes construyeron sus propuestas en torno a una pregunta problemática que debía explicarse con una teoría ya existente; los estudiantes de Negocios Internacionales utilizaron a Alvin y Heidi Toffler y La revolución de la riqueza, mientras que los estudiantes de política comercial y acuerdos internacionales, la sustentaron con la integración económica.

Todos participaron en el concurso y presentaron un stand sobre el país elegido y en donde explicaron la respuesta a su pregunta con muchos elementos característicos, trabajaron por grupos y la socialización se hizo a la comunidad 
universitaria, donde presentaron su ponencia en formato especial al jurado. Los estudiantes manifestaron "gran pánico al hablar frente al auditorio", pero lograron realizar una buena presentación gracias a diferentes ayudas audiovisuales, sin embargo y a pesar de los resultados, el público no los encontró satisfactorios para el desarrollo del proceso, aunque fue muy gratificante ver que estudiantes de primer y cuarto semestre podían desarrollar ponencias, manejar un auditorio y aprender a consultar para hacer una investigación, entre otras actividades. Finalmente, los estudiantes y la docente determinaron un alto compromiso desde el trabajo en equipo, con una representativa evolución cognitiva y un gran valor competitivo adquirido en el proceso.

El tercer proyecto fue diseñado para estudiantes de comercio internacional, política comercial y acuerdos internacionales de segundo y tercer semestre; en este espacio ellos debían seguir la estructura investigativa creando una ficha durante todo el semestre que mostrara la teoría en la práctica. La estructura a seguir fue: pregunta problémica, teoría que utilizaría el estudiante, desarrollo, experimento por medio de estadística y conclusiones.

Este trabajo lo debían convertir en un juego y presentarlo a la comunidad en el Casino Tagaste. Los estudiantes presentaron gran creatividad para desarrollar sus juegos, el desempeño y su compromiso con el conocimiento para que los invitados aprendieran el tema que presentaban de forma lúdica (experiencia personal, interdependencia, consciencia colectiva, entorno interno y externo). Dentro de los juegos usados por ellos podemos destacar twister, ruleta, tabla bailabre, escalera gigante, pequeña, Wii bolos, goloza, parques a la colombiana, pirinola, black jack, bolos, bingo, ruleta canadiense, cazadores de rompecabezas, dardo bomba, ruleta suiza, apuestas africanas y traga monedas, entre otros. Así lograron transformar el espacio en un casino de aprendizaje, todo esto gracias a que existió trabajo en equipo, ayuda, colaboración, búsqueda del bien común y práctica en las formas de resolución de problemas. En total participaron 100 estudiantes con un promedio de 4.5 sobre una calificación de 5.0.

Las calificaciones de estos proyectos se dieron teniendo como referencia las disincronías del desarrollo humano, es decir, que la obtención de la «meta» se debió al trabajo por el desarrollo armónico e integral de seis dimensiones o áreas de desarrollo de los estudiantes, sin que esto signifique que estas sean las únicas dimensiones, pero sí, las que fortalecen el proceso de enseñanzaaprendizaje. Estas dimensiones son: dimensión cuerpo-mente (relación años con evolución cerebral), psico-afectiva (procesos psicológicos), cognitiva (tipos de inteligencia y coeficiente intelectual), social-comunicativa (relación con su entorno), valorativa (moral, ética y práctica agustiniana) y de la praxis (cómo trasladar la teoría a la realidad).

Como resultado del anterior proceso se abrieron las convocatorias para crear un semillero de investigación. Finalmente, con 12 personas se consolidó el grupo 
Visionarios Emergentes, quienes han creado lazos de amistad y familiaridad, han participado en la ponencia de semilleros con una presentación lúdica, además de un artículo periodístico en la Universidad y un concurso de la Organización Mundial del Comercio. Este grupo sigue luchando por su semillero, teniendo un gran sentido de identidad y comprometiéndose cada vez más con sus trabajos, al mejorar en aspectos como el consultar, escribir, interpretar, reconocer el proceso de investigación, el estudio de las normas APA y su evolucionar como investigadores.

Las reuniones del grupo se han dado tanto virtual como presencialmente los sábados. Debido a que los integrantes tienen edades, características y puntos de vista particulares y pertenecen a diferentes semestres, es normal que existan discusiones y debates, los cuales han fortalecido, en gran medida, el trabajo en grupo del semillero.

\section{Conclusión}

Para lograr el trabajo del currículo visible y oculto ${ }^{4}$ fue necesario generar esta estrategia pedagógica, que se convierte en herramienta para la consecución de los objetivos, como es el caso de los proyectos de aula interdisciplinarios, donde se espera construir la transversalidad para permitir y consolidar un modelo del proceso de enseñanza-aprendizaje integrando espacios académicos, semestres, personas y grupos de personas en torno a una pregunta problémica sobre un tema determinado.

Al retomar las habilidades para la vida, planteadas por la Organización Mundial de la Salud, en los proyectos antes mencionados, se lograron desarrollar aspectos como el autoconocimiento, la empatía, la comunicación asertiva, las relaciones interpersonales, la toma de decisiones, la solución de problemas y conflictos, el pensamiento creativo, el pensamiento crítico, el manejo de emociones y sentimientos y el manejo de tensiones y estrés. 


\section{Referencias bibliográficas}

Bencomo, T.2007.Desarrollo de las TICs y la formación profesional. Visión gerencial. Vol. 6 (2): 163-184. Disponible en: http://www.saber.ula.ve/bitstream/123456789/25149/2/ articulo1.pdf. (1 octubre de 2012)

Cabrera, I. \& Vázquez. J. 2012. La Educación, un fenómeno social complejo. En Revista Digital Sociedad de la Información, $\mathrm{N}^{\circ} 38,2012$. http://www.sociedadelainformacion. com/38/complejo.pdf

CIAPA. 2012. Glosario. Disponible en: http://www.fapagaldos.com/index.php? option=com_content\&view=article\&id=238:glosario-de-terminos \&catid=81:glosario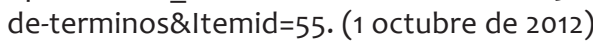

Chinoy, E. 1990. La sociedad: una introducción a la sociología. México: Ed. Porrúa, S. A.

Jurado, F. 2009. La educación básica y media en el Distrito Capital: orientaciones para la reorganización de la enseñanza por ciclos. Bogotá: Secretaría de Educación Distrital.

Malinowski, B. 1978. Una teoría de la cultura. Buenos Aires: Sudamericana.

Mosquera, R. 2002. Globalización y Alca, América para los americanos. Bogotá: Universidad Nacional de Colombia. Facultad de Ciencias Económicas.

Patiño, J. 2011. El trípode existencial de la propuesta pedagógica Agustiniana. Bogotá. Disponible en: http://www.uniagustiniana.edu.co/documentos/Tripode_Existencial. pdf. (1 octubre de 2012)

Radcliffe, A. 1975. El método de la antropología social. Barcelona: Anagrama.

Ruíz. E. 2000. Indicaciones para la presentación de proyectos. Bogotá: Agencia Colombiana de Cooperación Internacional.

Smith, A. 2010. La Riqueza de las Naciones. Recuperado de: http://stolpkin.net/spip. php?article696 (29 de noviembre de 2012).

Talcott, P. 1966. El sistema social. Revista de Occidente (1) p. 20.

Universitaria Agustiniana, 2012. El Trípode Existencial de la Propuesta Pedagógica de la Universitaria Agustiniana. Disponible en: http://www.uniagustiniana.edu.co/ documentos/Tripode_Existencial.pdf. (1 de octubre de 2012).

Vázquez, A. 2013. Incidencia de la brecha digital en grupos de iguales a partir de la interactividad entre la identidad física y la identidad digital. Tesis Doctoral. European Scientific Institute. Recuperado de: http://eujournal.org/files/journals/1/books/ AlfonsoVazquezAtochero.pdf (1 de enero de 2013). 